\title{
ELASTIC ANALYSIS OF POLYMERIC CYLINDERS REINFORCED WITH CARBON NANOTUBES
}

\author{
Hadi Hosseini, Reza Kolahchi, Mohammad Mehdi Heydari \\ Department of Civil Engineering, Khomein Branch, Islamic Azad University \\ Khomein, Iran \\ r.kolahchi@gmail.com
}

\begin{abstract}
In this work, stress analysis of a polymeric cylinder's cylindrical shell reinforced with carbon nanotubes (CNTs) is investigated. The cylinder is subjected to mechanical and thermal loadings. All the equivalent mechanical and thermal properties of nano-composite cylinder are obtained using Mori-Tanaka model. Based on equilibrium, the governing equation is derived. Using an exact solution, the stresses and radial displacement distributions in the structure are calculated. The effects of CNTs volume percent on the stresses and radial displacement for internal and external pressure cylinder are the main discussion of this study. Numerical results indicate that with increasing CNTs volume percent, the stresses decrease. However, this shows the important effect of nanotechnology in stress reduction of cylinder which may be useful in sensors and actuators.
\end{abstract}

Keywords: CNT, cylinder, elastic analysis, Mori-Tanaka model

\section{Introduction}

Composites offer advantageous characteristics of different materials with qualities that none of the constituents possess. Nano-composites developed in recent years have received much attention amongst researchers due to provision of new properties and exploiting unique synergism between materials. It has therefore been found multiple applications of nanocomposites in a wide range of industries including oil and gas, petrochemical, wire and cable, electronics, automotive, and construction.

Stress analysis of structures has been done by many researchers. Mechanical and thermal stresses in a functionally graded hollow cylinder were investigated by Jabbari et al. [1]. Analysis of the thermal stress behavior of functionally graded hollow circular cylinders was presented by Liew et al. [2]. You et al. [3] presented elastic analysis of internally pressurized thick-walled spherical pressure vessels of functionally graded materials. Dai et al. [4] studied exact solutions for functionally graded pressure vessels in a uniform magnetic field. Coupled thermoelasticity of functionally graded cylindrical shells was developed by Bahtui and Eslami [5]. 
Recently Ghorbanpour Arani et al. [6] investigated the effect of material in-homogeneity on electro-thermo-mechanical behaviors of functionally graded piezoelectric rotating shaft. Also, they [7] studied electro-thermo-mechanical behaviors of FGPM spheres using analytical method and ANSYS software.

With respect to developmental works on analysis of the cylinders, it should be noted that none of the research mentioned above have considered composite structure and their specific characteristics. Singh and Darip [8] analyzed nonlinear vibration of composite skew plate using a four-noded shear flexible quadrilateral high precision plate bending element. Nonlinear free vibration analysis of laminated composite skew thin plates was reported by Malekzadeh [9] using DQM. Qu et al. [10] employed Rayleigh-Ritz method for vibration analyzing of a damaged piezoelectric composite plate with different boundary conditions. Zhang et al. [11] presented a three-dimensional layerwise differential quadrature (DQ) method to analyze the linear laminated piezoelastic composite plates with different materials and boundary conditions. Thinh and Ngoc [12] carried out static behavior and vibration control of piezoelectric cantilever composite plates and comparison with experiments using FE method based on the first-order shear deformation theory. Using FE method, free vibration of laminated piezoelectric composite plates based on an accurate theory was studied by Shu [13]. Using Galerkin method, Shooshtari and Razavi [14] introduced a closed form solution for linear and nonlinear free vibrations of simply supported composite and fiber metal laminated rectangular plates based on the first order shear deformation theory. Nonlinear free vibration of geometrically piezoelectric laminated composite plate was studied by Dash and Singh [15] in the Green-Lagrange sense. Electro-thermo-mechanical nonlinear vibration and instability of a fluid conveying smart composite microtube made of polyvinylidene fluoride (PVDF) were investigated by Ghorbanpour Arani et al. [16] based on the modified couple stress theory and Timoshenko beam model.

In this work, volume percent of CNTs effect is studied on thermo-elastic analysis of nano-composite cylinders. The cylinder is subjected to mechanical and thermal loads. The governing equation is solved analytically for obtaining the stresses and radial displacement.

\section{Governing equations}

The components of displacement are assumed as

$$
u_{r}=u(r), u_{z}=u_{\theta}=0 .
$$

The equation of equilibrium is

$$
\frac{\partial \sigma_{r r}}{\partial r}+\frac{\sigma_{r r}-\sigma_{\theta \theta}}{r}=0,
$$


where $\sigma_{i i}(i=r, \theta)$ is the stress tensor. The corresponding constitutive relations may be written as [17]

$$
\begin{aligned}
& \sigma_{r r}=C_{11}\left(\varepsilon_{r r}-\alpha_{r r} T(r)\right)+C_{12}\left(\varepsilon_{\theta \theta}-\alpha_{\theta \theta} T(r)\right), \\
& \sigma_{\theta \theta}=C_{12}\left(\varepsilon_{r r}-\alpha_{r r} T(r)\right)+C_{22}\left(\varepsilon_{\theta \theta}-\alpha_{\theta \theta} T(r)\right),
\end{aligned}
$$

where radial and circumferential strains are therefore reduced to

$$
\begin{gathered}
\varepsilon_{r r}=\frac{\partial u}{\partial r}, \\
\varepsilon_{\theta \theta}=\frac{u}{r},
\end{gathered}
$$

Furthermore, the temperature distribution may be written as [17]

$$
T(r)=F_{1} \ln (\mathrm{r})+\mathrm{F}_{2},
$$

To develop the analytical solution, the following dimensionless quantities are introduced:

$$
\begin{aligned}
& \sigma_{r}=\frac{\sigma_{r r}}{C_{22}}, \quad \sigma_{\theta}=\frac{\sigma_{\theta \theta}}{C_{22}}, \quad \sigma_{z}=\frac{\sigma_{z z}}{C_{22}}, \quad U=\frac{u}{a}, \quad \chi=\frac{r}{a}, \beta=\frac{b}{a}, \\
& C_{1}=\frac{C_{11}}{C_{22}}, C_{3}=\frac{C_{13}}{C_{22}}, C_{4}=\frac{C_{33}}{C_{22}}, C_{2}=\frac{C_{12}}{C_{22}}, \bar{H}_{Z}=\frac{\mu H_{z}^{2}}{C_{22}}
\end{aligned}
$$

Using these notations and analytical method based on [17] yields the following in-homogeneity ordinary differential equations:

$$
\begin{aligned}
& R^{2} \frac{d^{2} \bar{u}}{d R^{2}}+R \frac{d \bar{u}}{d R}+\left(\frac{-1}{C_{1}}\right) \bar{u}=-\frac{-F_{2}\left(C_{2} \alpha_{\theta}+C_{2} \alpha_{r}+C_{1} \alpha_{r}-\alpha_{\theta}\right)-F_{1}\left(C_{2} \alpha_{\theta}+C_{1} \alpha_{r}\right)}{C_{1}} R \\
& -\frac{F_{1}\left(\alpha_{\theta}-C_{2} \alpha_{\theta r}-C_{1} \alpha_{r}\right)}{C_{1}} R \ln (R) .
\end{aligned}
$$

Equation (9), a non-homogeneous second-order ordinary differential equation, is the governing (constitutive) equation for displacement of the structure subjected to axisymmetric thermo-mechanical. The corresponding general solution for the homogeneous differential equation can be written as

$$
U_{g}=F_{4} \underbrace{\chi^{\Gamma_{1}}}_{u_{g 1}}+F_{5} \underbrace{\chi^{\Gamma_{2}}}_{u_{g 2}},
$$


in which $F_{4}$ and $F_{5}$ are integration constants to be determined by the boundary conditions. $\Gamma_{1}$ and $\Gamma_{2}$ are the roots of the corresponding characteristic equation of Eq. (9) and may be evaluated from

$$
\Gamma_{1,2}=\frac{\left(1-L_{1}\right) \pm \sqrt{\left(L_{1}-1\right)^{2}-4 L_{2}}}{2}
$$

The particular solution for Eq. (9) is assumed to have the form

$$
U_{p}=\chi^{\Gamma_{1}} U_{p 1}+\chi^{\Gamma_{2}} U_{p 2},
$$

where

$$
\begin{aligned}
& U_{p 1}=-\int \frac{\chi^{\Gamma_{2}} R(\chi)}{W_{p}} d \chi, \\
& U_{p 2}=\int \frac{\chi^{\Gamma_{1}} R(\chi)}{W_{p}} d \chi,
\end{aligned}
$$

where

$$
\begin{gathered}
W_{p}=\left[\begin{array}{cc}
U_{g 1} & U_{g 2} \\
\left(U_{g 1}\right)^{\prime} & \left(U_{g 2}\right)^{\prime}
\end{array}\right], \\
R(\chi)=-L_{3} \chi-L_{4} \chi \ln (\chi)-L_{5} .
\end{gathered}
$$

Finally, the radial displacement is

$$
U=U_{g}+U_{p} .
$$

Substituting $U$ from Eq. (17) into Eqs. (3) and (4), expressions for radial and circumferential stresses can be obtained. The boundary conditions for each case can be written as follows:

$$
\sigma_{r}(1)=-1, \quad \sigma_{r}(\chi)=-1, T(1)=1, T(\chi)=b .
$$

\section{Mori-Tanaka model and agglomeration effects}

In this section, the effective modulus of the composite shell reinforced by CNTs is developed. Different methods are available to estimate the overall properties of a composite [18]. Due to its simplicity and accuracy even at high volume frac- 
tions of the inclusions, the Mori-Tanaka method [18] is employed in this section. To begin with, the CNTs are assumed to be aligned and straight with the dispersion of uniform in the polymer. The matrix is assumed to be elastic and isotropic, with the Young's modulus $E_{m}$ and the Poisson's ratio $v_{m}$. The constitutive relations for a layer of the composite with the principal axes parallel to the $r, \theta$ and $z$ directions are [18]:

$$
\left\{\begin{array}{l}
\sigma_{11} \\
\sigma_{22} \\
\sigma_{33} \\
\sigma_{23} \\
\sigma_{13} \\
\sigma_{12}
\end{array}\right\}=\left[\begin{array}{cccccc}
k+m & l & k-m & 0 & 0 & 0 \\
l & n & l & 0 & 0 & 0 \\
k-m & l & k+m & 0 & 0 & 0 \\
0 & 0 & 0 & p & 0 & 0 \\
0 & 0 & 0 & 0 & m & 0 \\
0 & 0 & 0 & 0 & 0 & p
\end{array}\right]\left\{\begin{array}{c}
\varepsilon_{11} \\
\varepsilon_{22} \\
\varepsilon_{33} \\
\gamma_{23} \\
\gamma_{13} \\
\gamma_{12}
\end{array}\right\}
$$

where $\sigma_{i j}, \varepsilon_{i j}, \gamma_{i j}, k, m, n, l, p$ are the stress components, the strain components and the stiffness coefficients, respectively. According to the Mori-Tanaka method the stiffness coefficients are given by [18]:

$$
\begin{aligned}
& k=\frac{E_{m}\left\{E_{m} c_{m}+2 k_{r}\left(1+v_{m}\right)\left[1+c_{r}\left(1-2 v_{m}\right)\right]\right\}}{2\left(1+v_{m}\right)\left[E_{m}\left(1+c_{r}-2 v_{m}\right)+2 c_{m} k_{r}\left(1-v_{m}-2 v_{m}^{2}\right)\right]} \\
& l=\frac{E_{m}\left\{c_{m} v_{m}\left[E_{m}+2 k_{r}\left(1+v_{m}\right)\right]+2 c_{r} l_{r}\left(1-v_{m}^{2}\right)\right\}}{\left(1+v_{m}\right)\left[E_{m}\left(1+c_{r}-2 v_{m}\right)+2 c_{m} k_{r}\left(1-v_{m}-2 v_{m}^{2}\right)\right]} \\
& n=\frac{E_{m}^{2} c_{m}\left(1+c_{r}-c_{m} v_{m}\right)+2 c_{m} c_{r}\left(k_{r} n_{r}-l_{r}^{2}\right)\left(1+v_{m}\right)^{2}\left(1-2 v_{m}\right)}{\left(1+v_{m}\right)\left[E_{m}\left(1+c_{r}-2 v_{m}\right)+2 c_{m} k_{r}\left(1-v_{m}-2 v_{m}^{2}\right)\right]} \\
& +\frac{E_{m}\left[2 c_{m}^{2} k_{r}\left(1-v_{m}\right)+c_{r} n_{r}\left(1+c_{r}-2 v_{m}\right)-4 c_{m} l_{r} v_{m}\right]}{E_{m}\left(1+c_{r}-2 v_{m}\right)+2 c_{m} k_{r}\left(1-v_{m}-2 v_{m}^{2}\right)} \\
& p=\frac{E_{m}\left[E_{m} c_{m}+2 p_{r}\left(1+v_{m}\right)\left(1+c_{r}\right)\right]}{2\left(1+v_{m}\right)\left[E_{m}\left(1+c_{r}\right)+2 c_{m} p_{r}\left(1+v_{m}\right)\right]} \\
& m=\frac{E_{m}\left[E_{m} c_{m}+2 m_{r}\left(1+v_{m}\right)\left(3+c_{r}-4 v_{m}\right)\right]}{2\left(1+v_{m}\right)\left\{E_{m}\left[c_{m}+4 c_{r}\left(1-v_{m}\right)\right]+2 c_{m} m_{r}\left(3-v_{m}-4 v_{m}^{2}\right)\right\}}
\end{aligned}
$$

where $C_{m}$ and $C_{r}$ are the volume fractions of the matrix and the CNTs, respectively, and $k_{r}, l_{r}, n_{r}, p_{r}, m_{r}$ are the Hills elastic modulus for the CNTs [15].

\section{Numerical results and discussion}

In this section, the stress and radial displacement distribution are plotted for different values of CNT value percent. 
Figure 1 illustrates the effect of volume percent of CNTs $\left(C_{r}\right)$ on the radial stress of nano-composite cylinder. This figure indicates clearly that the volume percent of CNTs has a major effect on the radial stress. As can be seen, the radial stresses at the internal and external surfaces of the cylinder satisfy the given boundary conditions. Moreover, an increase in volume percent of CNTs results in increasing radial stress.

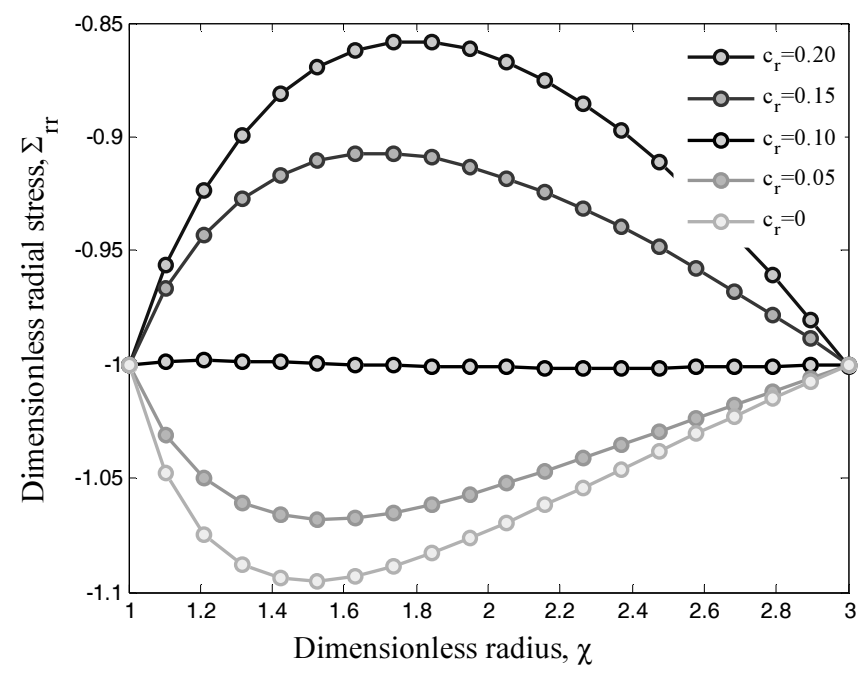

Fig. 1. Volume percent of CNT on the radial stress of structure

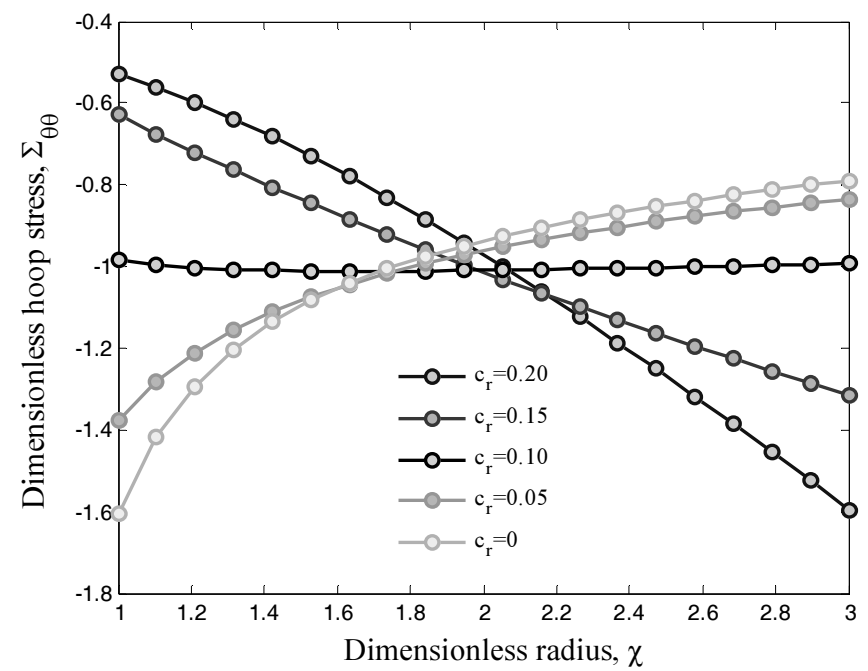

Fig. 2. Volume percent of CNT on the hoop stress of structure

The distributions of the hoop and effective stresses for different values of $C_{r}$ are displayed in Figures 2 and 3, where the hoop stress is increased from the inner 
to the outer radius for different values of $C_{r}$. Furthermore, the hoop and effective stresses decrease with increasing volume percent of CNTs. It is due to the fact that with increasing volume percent of CNTs, the cross-section of cylinder reduces and hence the more CNTs participate in load transfer.

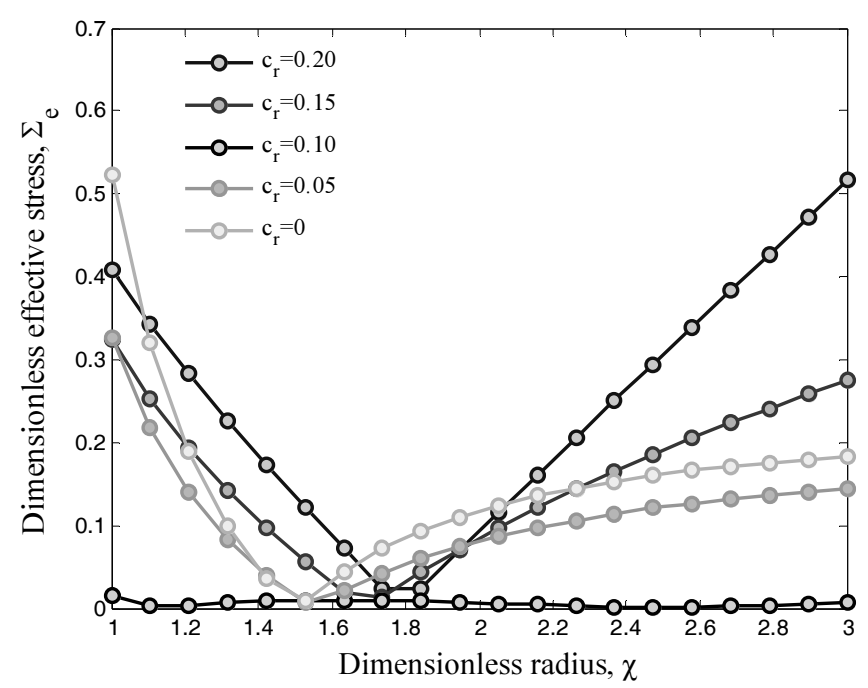

Fig. 3. Volume percent of CNT on the effective stress of structure

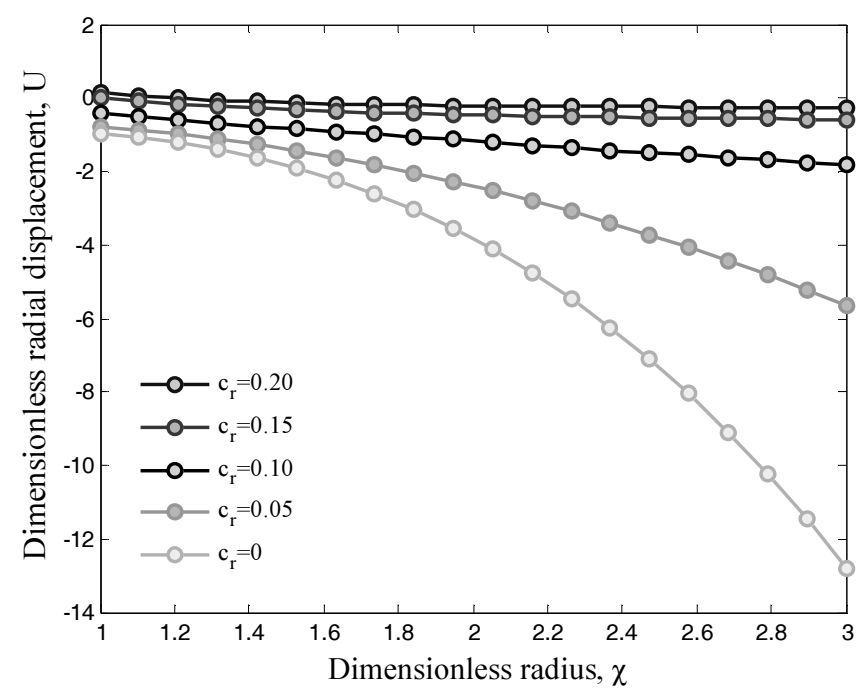

Fig. 4. Volume percent of CNT on the radial displacement of structure

The variations of the radial displacement along the radius for different values of volume percent of CNTs are demonstrated in Figure 4, which indicates that the radial displacement decreases with increasing $C_{r}$ and its maximum value 
occurs at the outer radius where the radial stress is maximum. It is because that with increasing volume percent of CNTs, the stability of structure increases and hence the radial displacement decreases. Moreover, the radial displacement tends asymptotically to a constant value throughout the wall thickness for $C=0.1$.

\section{Conclusions}

Volume percent of CNTs effect on the stresses and radial displacement distribution of a nano-composite cylinder is the main contribution of this work. The Mori-Tanaka model is used for calculating the equivalent material properties of system. An exact solution is applied for obtaining the stress and radial displacement of cylinder. Results indicate that with increasing volume percent of carbon nanotube, the stresses and radial displacement reduce. Moreover, the radial displacement tends asymptotically to a constant value throughout the wall thickness for all values of $C_{r}$. In addition, the volume percent of $C_{r}=0.1$ is optimized values since the distribution of stress become constant.

\section{References}

[1] Jabbari M., Sohrabpour S., Eslamic M.R., Mechanical and thermal stresses in a functionally graded hollow cylinder due to radially symmetric loads, Int. J. Press. Ves. Pip. 2002, 79, 493-497.

[2] Liew K.M., Kitipornchai S., Zhang X.Z., Lim C.W., Analysis of the thermal stress behaviour of functionally graded hollow circular cylinders, Int. J. Solids Struct. 2003, 40, 2355-2380.

[3] You L.H., Zhang J.J., You X.Y., Elastic analysis of internally pressurized thick-walled spherical pressure vessels of functionally graded materials, Int. J. Press Ves. Pip. 2005, 82, 347-354.

[4] Dai H.L., Fu Y.M., Dong Z.M., Exact solutions for functionally graded pressure vessels in a uniform magnetic field, Int. J. Solids Struct. 2006, 43, 5570-5580.

[5] Bahtui A., Eslami M.R., Coupled thermoelasticity of functionally graded cylindrical shells, Mech. Res. Commun. 2007, 34, 1-18.

[6] Ghorbanpour Arani A., Kolahchi R., Mosallaie Barzoki A.A., Effect of material in-homogeneity on electro-thermo-mechanical behaviors of functionally graded piezoelectric rotating shaft, Appl. Math. Model. 2011, 35, 2771-2789.

[7] Ghorbanpour Arani A., Kolahchi R., Mosallaie Barzoki A.A., Loghman A., Electro-thermomechanical behaviors of FGPM spheres using analytical method and ANSYS software, Appl. Math. Model. 2011, 36, 139-157.

[8] Singh M.K., Darip R., Nonlinear vibration of symmetrically laminated composite skewplates by finite element method, Int. J. Non Linear Mech. 2007, 42, 1144-1152.

[9] Malekzadeh P., A differential quadrature nonlinear free vibration analysis of laminated composite skew thin plates, Thin-wall Struct. 2007, 45, 237-250.

[10] Qu G.M., Li Y.Y., Cheng L., Wang B., Vibration analysis of a piezoelectric composite plate with cracks, Compos. Struct. 2006, 72, 111-118.

[11] Zhang Z., Feng C., Liew K.M., Three-dimensional vibration analysis of multilayered piezoelectric composite plates, Int. J. Eng. Sci. 2006, 44, 397-408. 
[12] Thinh T.I., Ngoc L.K., Static behavior and vibration control of piezoelectric cantilever composite plates and comparison with experiments, Comput. Mater. Sci. 2010, 49, 276-280.

[13] Shu X., Free vibration of laminated piezoelectric composite plates based on an accurate theory, Compos. Struct. 2005, 67, 375-382.

[14] Shooshtari A., Razavi S., A closed form solution for linear and nonlinear free vibrations of composite and fiber metal laminated rectangular plates, Compos. Struct. 2010, 92, 2663-2675.

[15] Dash P., Singh B.N., Nonlinear free vibration of piezoelectric laminated composite plate, Finite Elem. Anal. Des. 2009, 45, 686-694.

[16] Ghorbanpour Arani A., Abdollahian M., Kolahchi R., Nonlinear vibration of embedded smart composite microtube conveying fluid based on modified couple stress theory, Poly. Compos. 2015, 36, 1314-1324.

[17] Ghorbanpour Arani A., Kolahchi R., Mosallaie Barzoki A.A., Effect of material in-homogeneity on electromechanical behaviors of functionally graded piezoelectric rotating shaft, Appl. Math. Model. 2011, 135, 2771-2789.

[18] Mori T., Tanaka K., Average stress in matrix and average elastic energy of materials with misfitting inclusions, Acta Metall. Mater. 1973, 21, 571-574. 